\title{
Effects of different types of RSS data on the system accuracy of indoor localization system
}

\begin{abstract}
Indoor localization system becomes a substantial issue in recent research, especially in terms of the accuracy. Location based services have been used in many mobile applications as well as wireless sensor networks. High accuracy and fast convergence are very important issues for a good localization system. However, the type of obtained received signal strength (RSS) data is very important in order to get high accuracy. In this paper, we introduce three types of RSS data, which are: measured RSS, simulated RSS and average combined RSS. Bayesian network based on fingerprinting technique is used to investigate the effect of the three different types of RSS. The results show the effect of the three different RSS data on the accuracy of estimated location. The measured RSS has achieved an average accuracy of 4.3 meters using 10 training points while the average combined RSS has achieved a good accuracy of 2.1 meters.
\end{abstract}

Keyword: Bayesian network; Fingerprinting technique; Received signal strength 